INPLASY

PROTOCOL

To cite: Yang et al. Traditional Chinese medicine on treating myelosuppression after chemotherapy A protocol for systematic review and metaanalysis. Inplasy protocol

2020120097. doi:

10.37766/inplasy2020.12.0097

Received: 19 December 2020

Published: 19 December 2020

Corresponding author:

Songshan Liu

372193511@qq.com

Author Affiliation:

Hospital of Chengdu University

of Traditional Chinese

Medicine

Support: Fund of CDUTCM(No. ZRQN1744).

Review Stage at time of this submission: Preliminary

searches.

Conflicts of interest:

None.

\section{Traditional Chinese medicine on treating myelosuppression after chemotherapy A protocol for systematic review and meta-analysis}

Yang, S1; Che, H²; Xiao, L3; Zhao, B4; Liu, S5.

Review question / Objective: The study is aim to evaluate the efficacy and safety of the treatment that Chinese herbal medicine for myelosuppression after chemotherapy.

Condition being studied: Myelosuppression after chemotherapy is a common adverse reaction in the process of chemotherapy, mainly manifested as anemia, increased risk of bleeding, infection, the results seriously affect the quality of life and prognosis of patients, become the main cause of death. Since ancient times, traditional Chinese medicine (TCM) has been widely used in East Asia (such as China, Japan, South Korea) in the clinical treatment of bone marrow suppression after chemotherapy, which plays the role of synergism, toxicity reduction, immune regulation, and gradually developed into an indispensable role. Therefore, the purpose of this study was to use a net-work meta-analysis (NMA) to evaluate the evidence that traditional Chinese medicine is related to the efficacy and safety of chemotherapy-induced myelosuppression.

INPLASY registration number: This protocol was registered with the International Platform of Registered Systematic Review and Meta-Analysis Protocols (INPLASY) on 19 December 2020 and was last updated on 19 December 2020 (registration number INPLASY2020120097).

\title{
INTRODUCTION
}

Review question / Objective: The study is aim to evaluate the efficacy and safety of the treatment that Chinese herbal medicine for myelosuppression after chemotherapy.
Condition being studied: Myelosuppression after chemotherapy is a common adverse reaction in the process of chemotherapy, mainly manifested as anemia, increased risk of bleeding, infection, the results seriously affect the quality of life and 
prognosis of patients, become the main cause of death. Since ancient times, traditional Chinese medicine (TCM) has been widely used in East Asia (such as China, Japan, South Korea) in the clinical treatment of bone marrow suppression after chemotherapy, which plays the role of synergism, toxicity reduction, immune regulation, and gradually developed into an indispensable role. Therefore, the purpose of this study was to use a net-work metaanalysis (NMA) to evaluate the evidence that traditional Chinese medicine is related to the efficacy and safety of chemotherapyinduced myelosuppression.

\section{METHODS}

Search strategy: The following Chinese and English databases were searched electronically by keyword combination mode: PubMed, EMBASE and Cochrane Library, three British literature databases including PubMed, EMBASE and Cochrane Library, and four Chinese literature databases, including Chinese national knowledge infrastructure, VIP and Wanfang database, such as myelosuppression after chemotherapy and traditional Chinese medicine or medicinal plants or extracts of traditional Chinese medicine or traditional Chinese medicine formula or Chinese medicine Pharmaceutical preparations (TCM). We also limited the search time to December 2020 on the treatment of myelosuppression after chemotherapy with TCM.

Participant or population: All chemotherapy patients with myelosuppression after chemotherapy but not immediately lifethreatening will be included. This shows that the participants in our study will not have any gender, age or race, region and other restrictions.

Intervention: Choose TCM treatment that is not limited to dose, frequency, duration or follow-up time (including traditional Chinese medicine extract, traditional Chinese medicine preparation, single or mixed Chinese medicine formula, Chinese medicine combined with one or more Western Medicine). TCM intravenous injection, acupuncture, massage and other TCM treatment will be limited.

Comparator: For control intervention, patients who did not receive any treatment were regarded as blank control, and patients only treated by western medicine were treated as control intervention. In addition, once patients use TCM during the trial, they should immediately refuse.

Study designs to be included: This study will collect all randomized controlled trials (RCTs)based studies of TCM in the treatment of post chemotherapy myelosuppression, whether they have been published or not. Quasi-RCTs, non-RCTs, case reports and cross studies were not included in the collection.

Eligibility criteria: All chemotherapy patients with myelosuppression after chemotherapy but not immediately lifethreatening will be included. This shows that the participants in our study will not have any gender, age or race, region and other restrictions.

Information sources: The following Chinese and English databases were searched electronically by keyword combination mode: PubMed, EMBASE and Cochrane Library, three British literature databases including PubMed, EMBASE and Cochrane Library, and four Chinese literature databases, including Chinese national knowledge infrastructure, VIP and Wanfang database, such as myelosuppression after chemotherapy and traditional Chinese medicine or medicinal plants or extracts of traditional Chinese medicine or traditional Chinese medicine formula or Chinese medicine Pharmaceutical preparations (TCM). We also limited the search time to December 2020 on the treatment of myelosuppression after chemotherapy with TCM. We will also manually search for grey documents such as conference minutes, dissertations, and trial registration (through the WHO International Clinical Trial Registration Platform (ICRP) and the national registry website). 
Main outcome(s): The main outcome was the elevation of hemoglobin, platelets, leukocytes and neutrophils. The main outcome was the elevation of $\mathrm{Hb}$ and PLT and WBC.

Additional outcome(s): Clinical symptoms, Quality of life, Absolute value of RTC, Percentage of RTC, Low fluorescent RTC, Medium fluorescent RTC, High fluorescent RTC.

Data management: Literature data collection will be input by two researchers through EpiData 3.1 software, and the final data collection will be updated to the database by another researcher after checking the consistency. The contents of data extraction include: title, first author, contact information, diagnostic criteria of myelosuppression, age, gender, severity, duration of suppression, intervention measures used in experimental and control groups, treatment time of TCM, safety indicators and number of adverse reactions. If the research data is insufficient, unclear or missing, we will contact the first author of the study through contact information to improve the information. Those who failed were excluded from the study.

Quality assessment / Risk of bias analysis: The risk of bias in this study will be assessed from seven perspectives of Cochrane tool: sequence generation, assignment concealment, blinding participants, blinding testers, blinding result assessors, incomplete result data, and selective reporting of results. The above assessment will be completed by two well-trained research members. If there is disagreement, the problem can be solved through group discussion. If the opinions are still different, actively contact the author or consult a third assessor. We will also assess the evidence quality of the main results. The assessment includes five aspects: heterogeneity, indirectness, bias risk, imprecision and publication bias, and uses "low risk", "high risk" or "unclear risk of bias" to make corresponding judgments on each level of evidence.EPOC guidelines will be used to assess the risks of nonRCTs.

Strategy of data synthesis: Revman software (version 5.3.5) and $R$ software (version 3.6.1) will be used for protocol of meta in this paper. If possible, the analysis of all results will be done by intention to treat. We will conduct an analysis to provide estimates of the effect of dichotomy data and continuous data with a confidence interval of $95 \%$. For dichotomy data, we will use the risk ratio (RR) and the average difference (MD) of continuous data. We will explore heterogeneity before meta-analysis of the results. Standard chi square test was used to detect the heterogeneity, and the significance level was $p<0.10$. 12 statistics will be used to quantify the inconsistencies among studies and to assess the impact of heterogeneity on the meta-analysis. The results of dichotomy were obtained by mantel heinsell method, and the continuous results were obtained by de Simone and Laird inverse variance methods. Random effects models will be used to collect data.

Subgroup analysis: We will conduct subgroup and meta regression analysis. If enough studies are included (at least 10 trials), the aim is to explore the heterogeneity sources of myelosuppression in terms of classification, complications, Chinese medicine treatment course and treatment plan.

Sensibility analysis: We will report the results of sensitivity analysis by eliminating low-quality tests to evaluate the reliability and robustness of the results.

Language: Language is limited with English and Chinese.

\section{Country(ies) involved: China.}

Other relevant information: Evidence evaluation: We will assess the evidence quality of the main results through the proposed hierarchical assessment, development and evaluation methods. The assessment includes five aspects: 
heterogeneity, indirectness, bias risk, imprecision and publication bias, and uses "high", "moderate", "low" and "very low" to make corresponding judgments on each level of evidence.

Keywords: traditional Chinese medicine, myelosuppression after chemotherapy, blood corpuscle, clinical symptoms, quality of life, protocol, systematic review.

Dissemination plans: We will disseminate the results of this systematic review by publishing the manuscript in a peer reviewed journal.

Contributions of each author:

Author 1 - Sihang Yang.

Author 2 - Hong Che.

Author 3 - Li Xiao.

Author 4 - Bingjie Zhao.

Author 5 - Songshan Liu. 